PUPT-1779

hep-th/9803191

March 1998

\title{
DLCQ-M(atrix) Description of String Theory, and Supergravity.
}

\author{
Gilad Lifschytz \\ Department of Physics, Joseph Henry Laboratories, \\ Princeton University, \\ Princeton, NJ 08544, USA. \\ e-mail: Gilad@puhep1.princeton.edu
}

\begin{abstract}
We discuss the connection between Matrix string theory and the DLCQ of string theory. Using this connection we describe the sense in which perturbative string amplitudes are reproduced in the Matrix string theory. Using recent realization of the connection between SYM and Supergravity, we suggest how to describe Matrix theory with non-flat backgrounds.
\end{abstract}




\section{Introduction}

Recently Seiberg [1] considered the DLCQ formulation of M-theory, and showed that it is equivalent to the BFSS formulation of M(atrix) theory [2], as conjectured in [3]. The starting point is M-theory (or string theory), compactified on a vanishing small circle. M(atrix) theory (on $T^{d}$ ) is then seen

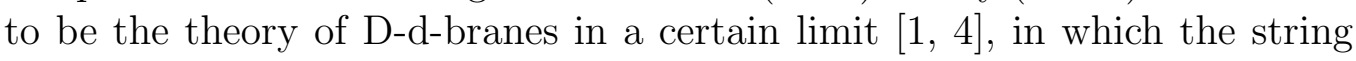
length and the string coupling are taken to zero but a certain combination of them stays fix.

What is the meaning of this limit?. The connection with DLCQ description comes up as we define the sector with $P_{-}=N / R$ of DLCQ as a limit of vanishing compact direction with $N$ units of momenta in this direction [5]. This can also be seen by formally boosting a theory in a compact direction and taking the radius of this direction to shrink thus defining a limiting procedure for null compactification.

More recently, using argument relating Black holes to D-branes, It was argued [6], using [7, 8, 9, 10], that conformal SYM theory properties can be calculated in a region where perturbation theory is not valid, by supergravity on a certain background. Similar argument for other SYM were given in [11]. The precise identification for the conformal SYM was given in [12, 13]. The limits taken in [6, 11] are the same as the ones taken for the DLCQ description.

One issue that needs to be addressed in DLCQ is the fate of states with $P_{-}=0$. One advantage of looking at DLCQ of string theory rather than Mtheory is the issue of these zero-modes, which tend to make the loops diverge in field theory [14. In the case of string theory this seems not to be the case 15]. Then it is possible to show that the SYM will reproduce perturbative tree level scattering amplitudes, in the appropriate limit. If one starts with a non-trivial backgrounds, as we will see, it can be incorporated into the SYM description of the DLCQ of string theory, using the recent connection with supergravity.

In section (2) we describe the M(atrix) description of Type IIA string theory [16, 17, 18] following Seiberg and Sen. We review the the degrees of freedom and the generalization to include certain backgrounds. In section (3) we discuss scattering amplitude, and give a precise correspondence which shows the emergence of perturbative amplitudes in the M(atrix) string, we then discuss the supergravity side and show that the existence of nonrenormalisation theorems needed for the $\mathrm{M}$ (atrix) theory to work are compatible with [6, 11]. In section (4) following [12, 13] we describe how the states with no momenta in the compact vanishing direction couple to the SYM. This gives a way to describe the effect of $P_{-}=0$ states, in particular the effect of non-flat backgrounds. 


\section{Type IIA string Theory}

We start with Type IIA string theory with string coupling $g_{s}$, a string length $l_{s}$, and a generic length $\mathrm{L}$. We now compactify it on a circle of radius $R_{s}$ (which will be taken to zero) and put $\mathrm{N}$ units of momenta along the circle. We will use a set of duality transformation to map it into another string theory. We first perform a T-duality along the circle this gives'

$$
\begin{aligned}
g_{s}^{\prime} & =g_{s} \frac{l_{s}}{R_{s}} \\
R_{s}^{\prime} & =\frac{l_{s}^{2}}{R_{s}} \\
L^{\prime} & =L
\end{aligned}
$$

The $\mathrm{N}$ units of momenta along the circle transform to $\mathrm{N}$ Type IIB winding strings. As the string coupling will become large we perform a S-duality. This gives

$$
\begin{aligned}
g_{s}^{\prime \prime} & =\frac{R_{s}}{g_{s} l_{s}^{2}} l_{s} \\
R_{s}^{\prime \prime} & =\frac{l_{s}^{2}}{R_{s}} \\
L^{\prime \prime} & =L \\
\left(l_{s}^{\prime \prime}\right)^{2} & =\frac{g_{s} l_{s}^{2}}{R_{s}} l_{s} .
\end{aligned}
$$

The string winding become D-string winding. Already we see that any generic length $L$ is much smaller than the string length. To get the SYM limit from the parameters above, we have to define a new scale. The SYM energies from the parameters in equation(2) are all proportional to $R_{s}$. One then just rescale all energy scale by a factor $\bar{R} / R_{s}$ where $\bar{R}$ can be anything. If however we choose $\bar{R}=R$ then the SYM description would match to the original starting point (DLCQ of Type IIA with parameter $R$ ), with no other scaling necessary. This means that all length scales change by $R_{s} / R$, including time. The parameters of the Type IIB theory are now

$$
\begin{aligned}
g_{s}^{n} & =\frac{R_{s}}{g_{s} l_{s}^{2}} l_{s} \\
R_{s}^{n} & =\frac{l_{s}^{2}}{R} \\
L^{n} & =L R_{s} / R \\
\left(l_{s}^{n}\right)^{2} & =R_{s} \frac{g_{s} l_{s}^{2}}{R^{2}} l_{s} .
\end{aligned}
$$

\footnotetext{
${ }^{1}$ Through out the paper we drop factors of $2 \pi$
} 
and one arrives at the SYM theory. The Yang-Mills coupling constant on the D-strings is

$$
g_{y m}^{2}=g_{s}^{n}\left(l_{s}^{n}\right)^{-2}=\frac{1}{g_{s}^{2}}\left(\frac{R}{l_{s}^{2}}\right)^{2}
$$

and the Higgs vev $L^{n} /\left(l_{s}^{n}\right)^{2}$ and $v t /\left(l_{s}^{n}\right)^{2}$ are finite.

In this limit the only surviving degrees of freedom are represented by SYM in $(1+1)$ dimensions on a circle. The SYM Hamiltonian then represents the lightcone energy. It is given by the total energy of the system minus the mass of the $N$ D-strings.

Equation (2) can be obtained also by starting with the original set up and instead of the $\mathrm{T}$ and S-dualities just exchange the coupling direction with the $R_{s}$ direction, using the M-theory interpretation. However the first path can lead to formulations of backgrounds without a known M-theory interpretation as we will see below.

If we have originally $k$ compact directions $L_{i}$ we now T-dualise in directions $L_{i}$ to get

$$
\begin{aligned}
g_{s}^{\prime \prime \prime \prime} & =\frac{R_{s}}{g_{s} l_{s}^{2}} l_{s} \prod_{i}^{k} \frac{l_{s}^{\prime \prime \prime \prime}}{L_{i}}=\left(\frac{R_{s}}{g_{s} l_{s}^{2}}\right)^{1-k / 2} \prod_{i} \frac{l_{s}}{L_{i}} l_{s}^{1-k / 2} \\
R_{s}^{\prime \prime \prime \prime} & =\frac{l_{s}^{2}}{R_{s}} \\
L_{i}^{\prime \prime \prime \prime} & =\frac{g_{s} l_{s}^{2}}{R_{s}} \frac{l_{s}}{L_{i}} \\
\left(l_{s}^{\prime \prime \prime \prime}\right)^{2} & =\frac{g_{s} l_{s}^{2}}{R_{s}} l_{s} .
\end{aligned}
$$

After scaling, the SYM coupling constant in $((1+\mathrm{k})+1)$ dimensions is then

$$
g_{y m}^{2}=\left(\frac{R}{g_{s} l_{s}^{2}}\right)^{2-k} \prod_{i} \frac{l_{s}}{L_{i}}
$$

\subsection{Degrees of freedom}

Let us now look back and try to be more precise about the detailed relationship between the Type IIA string theory and the SYM description 16, 17, 18]. The DLCQ description of the Type IIA is related under a Lorenz boost to the Type IIA on a vanishing small circle. This under T, and S-dualities is converted to a Type IIB theory of D-strings, as above. If we further scale the parameters we can see that we end up with SYM. On the Type IIA string theory side one has the massless modes (graviton, dilaton etc) and the massive modes of the string. On the Type IIB with D-strings side the massless modes are constructed from the fermionic zero-modes of the D-strings. Winding states around the vanishing circle are described by a state with mo-

menta along the direction the D-string is wrapped $\left(R_{s}^{\prime \prime}\right)$. The lowest unit of 
momenta is $1 / R_{s}^{\prime \prime}$, This is true even if the string is multiply wound. However on the D-string one can have states with net momenta zero, but energies

$2 / N R_{s}^{\prime \prime}$ [19], where $N$ is the wrapping number of the D-string. These are just states with open string running on both directions of the wrapped D-string, with momenta $\pm 1 / N R_{s}^{\prime \prime}$. These represent the massive states of the original string theory. This can be seen from following the dualities and from the lightcone energies

$$
E=\sqrt{\left(N / R_{s}\right)^{2}+l_{s}^{-2}}-N / R_{s} \sim R_{s} / N l_{s}^{2}
$$

From this one can see that in the SYM it cost less energy to produce a massive state than a winding state in the large $N$ limit. In a general scattering, these modes will be excited if there is enough energy as in (10). From this and the uncertainty relationship

$$
p_{\perp}^{\max } \sim 1 / b_{\min }
$$

one finds $b_{\min } \sim l_{s}$ which is expected from elementary string states.

The SYM description has more states than we have described. In particular it has the degrees of freedom which are the off diagonal entries of the matrices. What do these correspond in the original string theory. At weak coupling string theory we do not see them, but the SYM is T, and S-dual to a string theory on a small circle, for any string coupling. Indeed looking at a string theory with a small circle, one can exchange the coupling direction with the small circle direction $\left(R_{s}\right)$. Now the new string length is $l_{s}^{\prime \prime}$. There could be excitations of open membranes where the wrapped membrane wraps around $R_{s}$ and stretched between the two objects. This will have energy $\sim b /\left(l_{s}^{\prime \prime}\right)^{-2} \sim R_{s}$. Indeed these are the off diagonal terms. In order for these to be excited in a collision of massless states, one needs

$$
\frac{p_{\perp}^{2}}{2 M} \sim \frac{b R_{s}}{g_{s} l_{s}^{3}}
$$

Where $M=N / R_{s}$ is the D-string mass. Comparing equations (10, 12) we see that as long as $b / R_{11}$ is large the dominant excitations are the original massive string excitations and we are in the perturbative string regime. Once out of this regime we have more possible excitations, and in the M-theory limit the new excitations will dominate. When the stringy excitations are unexcitable, there is a new limit to the smallest distance one can probe, $b_{\text {min }} \sim l_{p}$ 20.

\subsection{Type IIA Backgrounds}

It is also of interest to derive DLCQ of Type IIA with some branes present. After the sequence of T-duality and S-duality these branes will become some other branes and the theory will then be modified from the SYM in $(1+1)$ 
dimensions to some other supersymmetric gauge theory that includes the effect of the extra branes. Notice however that if we end up after the dualities with a D-brane of dimension greater than one, the Yang-MIlls coupling constant on the brane will become zero in the limit $R_{s} \rightarrow 0$, thus its own excitations do not contribute. In addition the brane can be boosted along their world volume or orthogonal to it. If they are boosted along they do not pick up any momentum and eventually are wrapped on a circle of size $R_{s}$. In general any other string state will pick up momenta and their interaction with the brane will be represented in the gauge theory. This theory will have 8 supercharges. If we boost transverse to the brane it will pick up momenta that will be bound to it to form a BPS state. After the dualities the brane appears as a charge in SYM. In the following we give some examples.

- start with Type IIA with D0 branes. After the set of dualities we end up with D1 branes and fundamental strings in direction 1 The theory on the D1 branes is then SYM in a sector with background electric charge 18 .

- Start with $k$ NS-five brane with world volume directions $(0,1,2,3,4,5)$. We will take $R_{s}$ to be always in direction 1 . After the T, and S-dualities this becomes a D- 5 brane in directions $(0,1,2,3,4,5)$. The D- 1 branes are in direction 1 with that direction having length $R_{s}^{\prime \prime}$ as given above. The gauge theory Lagrangian includes other than the Lagrangian of SYM in $(1+1)$ dimensions $k$ extra hypermultiplets [21].

- Start with the D4-brane in directions $(0,1,2,3,4)$. After the set of dualities we get a D3-brane in directions $(0,2,3,4)$ and a D-1 brane in direction 1. Again the length of direction 1 is $R_{s}^{\prime \prime}$. If we are interested in the limit in which the D4-brane becomes an M-5 brane one should take the large $g_{s}$ limit. If one is interested in the flat M-5 brane limit then we can T-dualise to get the theory of D4 branes and D0 branes 22].

- Start with the D6-brane in direction $(0,1,2,3,4,5,6)$. After the dualities we get a NS-five brane in direction $(0,2,3,4,5,6)$ and a D1-brane in direction $1[23$.

- Start with a D8-brane in direction $(0,1,2,3,4,5,6,7,8)$. This does not have a known M-theory interpretation. This configuration is probably not a consistent type IIA configuration as the dilaton blows up a finite distance away from the D8 brane. Never the less let us look at the transformation. After the T-duality we end up with a D7 brane and winding, after a S-duality we get a $(0,1)$ seven brane in direction $(0,2,3,4,5,6,7,8)$, and $N$ D1 branes in direction 1 . If we had $k$ D8branes we can end up with $K(0,1)$ seven branes. The D1 branes can 
be outside the seven branes (that is separated in the 9 direction), or the D1 brane can break between the $(0,1)$ seven branes. The theory on the D1 branes will then give the matrix formulation of the type IIA theory in a massive background. The details of the gauge theory will be discussed elsewhere

\section{Scattering amplitudes}

In this section we will show that Seiberg prescription guarantees the correct scattering amplitudes with no $P_{-}$exchange, to be reproduced by the SYM. When one starts with a Type IIA on a small circle, it might be that loops become increasingly important as the circle shrinks. This however was shown not to be the case for the one loop [15], if the on shell particles have momenta in the compact direction, and it seems reasonable that this will hold to all loops?. This means that there is a good perturbative expansion for scattering amplitudes we are interested in, of Type IIA on a vanishing small circle. What we will see, is that all scattering amplitudes have a common factor of $R_{s}$ which is the expected one from the Lorenz transformation, and that after scaling, in the SYM it gives finite results. Using T and S-duality this means that tree amplitudes are reproduced in the SYM. It is important to note that while the tree amplitudes with no $P_{-}$exchange are the same as those for ten-dimensional Type IIA theory, for finite $N$, the loop correction while finite, are different then the ten-dimensional ones.

\subsection{Kinematics}

We consider scattering of ten dimensional massless particles. The massless states have momenta

$$
P^{i}=\left(\sqrt{\left(N_{i} / R_{s}\right)^{2}+\vec{p}_{i}^{2}}, N_{i} / R_{s}, \vec{p}_{i}\right) .
$$

All the kinematic variables $\left(P_{i}+P_{j}\right)^{2}$ are finite, and so are the term involving polarizations, this of course is a consequence of Lorenz invariant.

In order to compute S-matrix elements one has to normalize the states appropriately. In order to compare later to the SYM we chose the non relativistic normalization for the states. Each incoming and outgoing state gives a factor of $\frac{1}{\sqrt{2 P_{0}}}$ As one direction is compact with radius $R_{s}$ each of the incoming and outgoing states gives a factor of $\frac{1}{\sqrt{R_{s}}}$. In addition the delta function of the momentum in the compact direction becomes a $R_{s} \delta_{\left(\sum N_{i}, \sum N_{f}\right)}$. If The particles did not have any momenta in the compact direction then the $n$-point amplitude would have had an explicit factor of $R_{s}^{1-n / 2}$ which would

${ }^{2}$ The actual calculation in [15] was for a one loop four point amplitude, but the mechanism responsible for finiteness seem general 
have given an effective string coupling $g_{s} / \sqrt{R_{s}}$ as one expects from low energy considerations. In our case however $n$-point amplitudes just have a factor of $R_{s}$. Thus its Fourier transform (the potential) will also have a $R_{s}$ factor, which is what happens in the T-S-dual description. In both case the reason is the same. The time has an explicit $1 / R_{s}$ factor, picked up from the Lorenz transformation.

These Kinematic factors multiply the usual string amplitudes as computed for instance in [24]. The tree level amplitudes with no momentum exchange in the compact direction, do not know if there is a compact direction (other then through the normalization of states as discussed above), and are only a function of the Lorenz invariant variables. Thus they give the same result as if we had ten large dimensions, and this is true for any $N$. In the loops the zero-modes run also and the loops are slightly different than the ten-dimensional loops but are still finite [15]. The difference between the loop result and the ten dimensional loop result differs only in an overall factor depending on $N_{i}$, this may be the origin of the discrepancy found in [25, 26]. If we look for long distance results (or at small $g_{s}$ ) the tree level will be the leading term, and thus will be reproduced in the M(atrix) theory. It seems that in the large $R$ and large $N$ limit, the loops will also match and one will recover lightcone string theory in ten dimensions.

We would like to note that we do not actually know how to calculate these scattering amplitudes in SYM, and the discrepancy with the tree threegraviton scattering still needs to be solved [27], but given theses arguments it seem to be more of a technical problem than a real discrepancy.

\subsection{The Usual Example}

Let us look at the scattering of two massless states in the long distance limit. In the string theory for small momentum transfer one has

$$
A_{4} \sim g_{s}^{2} \frac{R_{s}}{N_{1} N_{2}} \frac{s^{2}}{t}
$$

where

$$
s=\left(P_{1}+P_{2}\right)^{2}=\frac{1}{2} N_{1} N_{2}\left(\frac{\overrightarrow{p_{1}}}{N_{1}}-\frac{\overrightarrow{p_{2}}}{N_{2}}\right)^{2} .
$$

If there is no change in $N$ of the scattered particles then

$$
t \sim\left(\vec{p}_{1}-\overrightarrow{p_{1}}\right)^{2}
$$

and one can easily define a non-relativistic like potential by Fourier transformation. If the particles after scattering have a different $N$, the amplitude is still well defined. In the large $N$ limit exchange of a unit of momenta in the compact direction will result in a very small change of $t$. 

by

On the D-string side the potential between two moving D-strings is given

$$
V \sim N_{1} N_{2} \frac{R_{s}^{\prime \prime}(\Delta v)^{4}\left(l_{s}^{\prime \prime}\right)^{4}}{r^{6}}
$$

using $v=\frac{\vec{p}}{N / R_{s}}$, and equation (2), this matches the elementary string result. To get the DLCQ result, after the Lorentz transformation, one has to multiply by another factor of $R$.

Notice that what we call $v^{4}$ term, comes from a Lorenz invariant expression $\left(s^{2}\right)$, and as such is Lorenz invariant [5].

\subsection{Supergravity Side}

We have started with a type IIA string theory on a small circle, with $N$ units of momenta along direction 1. The metric associated with a wave with $N$ units of momenta in a compact direction $R_{s}$ is given by

$$
\begin{aligned}
d s^{2} & =-d t^{2}(2-H)+d x_{1}^{2} H+2(H-1) d t d x_{1}+d x_{i} d x^{i} \\
H & =1+c \frac{g_{s}^{2} l_{s}^{8} N}{R_{s}^{2} r^{6}}
\end{aligned}
$$

where $c$ is a numerical constant. We would like to calculate the scattering of another particle, with momenta $n / R_{s}$ in direction 1 and transverse momenta $\vec{p}$, from the above metric, in the supergravity approximation. This can be done by evaluating the action of one particle in the presence of another, and one can find a finite term even in the limit $R_{s} \rightarrow 0$.

Instead of presenting the derivation in this fashion (see [29]) we would to do it in a way to make contact with recent developments. Let us follow the dualities, using the rules for $\mathrm{T}$ and S-duality [30], one gets the solution for D-strings,

$$
\begin{aligned}
d s^{2} & =H^{-1 / 2}\left(-d t^{2}+d x_{1}^{2}\right)+H^{1 / 2} d x_{i} d x^{i} \\
B_{01} & =-\left(H^{-1}-1\right) \\
e^{-\left(\phi-\phi_{\infty}\right)} & =H^{-1 / 2}
\end{aligned}
$$

where $e^{\phi_{\infty}}=g_{s}^{\prime \prime}$, and $x_{1}$ has periodicity $l_{s}^{2} / R_{s}$. In the limit $R_{s} \rightarrow 0$

$$
H \sim c \frac{N g_{s}^{2} l_{s}^{8}}{R_{s}^{2} r^{6}}
$$

and things again may look singular. This however represent the background fields that an original probe will feel, and as scattering of the momenta states (in string theory) was shown to be well define, one might expect a similar

\footnotetext{
${ }^{3}$ for Four graviton scattering in the orbifold limit of SYM see [28]
} 
thing to happen here (in the appropriate limit). To compute the long distance interaction, one couples the D-string to a background generated by equation (19), using the Born Infeld action, plus the WZW term.

A test D-string will couple through the action

$$
S=T_{2}\left(\int d^{2} \sigma e^{-\left(\phi-\phi_{\infty}\right)} \sqrt{\operatorname{det} G}+\int B^{R R}\right) .
$$

where $T_{2}=\frac{1}{g_{s}^{\prime \prime} l_{s}^{\prime \prime}}=l_{s}^{-2}$, is finite as $R_{s} \rightarrow 0$. We have suppressed here the dependence on the world volume gauge field of the probe, this can also be taken into account and will also produce finite results.

Taking a simple case where the test D-string is just rigidly moving with a velocity $v$, we evaluate the action (in static gauge) and find

$$
S=T_{2} \int d t d x_{1} H^{-1}\left(\sqrt{1+H v^{2}}-1\right)=\int d t\left[m v^{2}+V(v, r)\right]
$$

Now $H=c R_{s}^{-2} \frac{N g_{s}^{2} l_{s}^{6}}{r^{6}}$ but $v \sim R_{s}$ so inside the square root every thing is finite. In addition $x_{1}$ has a periodicity of $1 / R_{s}$ so after doing the $x_{1}$ integral the action $\sim R_{s}$. This gives a potential which is $\sim R_{s}$ as we have found before. Now one can scale all length scales by $R_{s} / R$ to get a finite potential. This potential (from equation (22)) coincides with the one conjectured to be the sum of planer diagrams in the SYM side [5, 29, 31, 32], and indeed checked up to two-loops. It is interesting to note that a probe anti-brane, will also produce a finite result for a potential.

To make contact with [11], start with equation (19) and scale all coordinates by a factor $R_{s} / R$. This gives the Type IIB the set of parameters of equation (3), and in this limit we have seen all SYM energies are finite. Now define $U=\frac{r R}{g_{s} l_{s}^{3}}$ and $\alpha=R_{s} \frac{g_{s} l_{s}^{2}}{R^{2}}=\left(l_{s}^{n}\right)^{2}$. Then the metric becomes

$$
d s^{2}=\alpha\left[\frac{U^{3}}{g_{y m} \sqrt{c N}}\left(-d t^{2}+d x_{1}^{2}\right)+\frac{g_{y m} \sqrt{c N}}{U^{3}}\left(d U^{2}+U^{2} d \Omega_{7}\right)\right] .
$$

which is of course the same one given in [11] (see also [34, 33]). Notice that here however $x_{1}$ is compact with period $l_{s}^{2} / R$, and that $t$ is not dilated any more.

What is the range of validity of this metric ?. The curvature of this solution is (in the original Type IIA variables) $\alpha^{2} R^{2} \sim \frac{r^{2}}{l_{s}^{2} N}$ while the local string coupling is $e^{2 \phi} \sim \frac{N l_{s}^{6}}{r^{6}}$. In addition because one has a compact direction it should be bigger than the string length giving the condition $\left(\frac{r}{l_{s}}\right)^{2} \gg g_{s}^{4 / 3} N^{1 / 3}$. From this one might expect the calculation given in equation (22) to be valid in the region (for small $g_{s}$ )

$$
N^{1 / 3} \ll \frac{r^{2}}{l_{s}^{2}} \ll N
$$


For large $g_{s}$ the left limit is replaced with $g_{s}^{4 / 3} N^{1 / 3}$.

On the other hand The SYM perturbative expansion, for this process, is valid if

$$
\frac{r^{2}}{l_{s}^{2}} \gg N
$$

Still particular terms in the SYM result seems to agree with the supergravity result. This was conjectured to be a consequence of non-renormalisation theorems on the SYM side, which would have to be true for M(atrix) theory to work. Given that one gets both the large $N$ SYM and supergravity limit as the same limit of parameters, one sees that indeed the non-renormalisation theorem are correct (see [29] for another argument).

One can look at the opposite side, why is it that the supergravity result seemed to be valid much beyond it's naive validity. one possibility is as follows. Usually if the curvature becomes large then one can not trust the classical solution, because higher curvature terms can change the solution. However the background that we started with was that of a plane-fronted wave, and the potential derived in equation (22) is the same as one could have derived from the plane fronted wave solution. This solution is known not to be corrected by higher curvature terms [35].

\section{Non-trivial backgrounds}

We started off with Type IIA on vanishing small circle of radius $R_{s}$. The states that we have been considering are those with positive momenta in the compact direction. These are the states that in the DLCQ have non vanishing $P_{-}$. The massless states with $P_{1}=0$ (which define a non-flat background) transform under $\mathrm{T}$ and $\mathrm{S}$-duality to be the massless elementary string states in the Type IIB with D-strings, picture. One would like to know how to encompass them in the SYM description.

As we have seen, the SYM that one ends up is Type IIB with D-strings and parameters given by equation(2). This limit is equivalent to a limit in which both the string length and the string coupling constant vanish (equation (3)). Still the metric generated by the D-strings although $\sim R_{s}$ gives finite results for scattering of another D-string (as in the previous section). As argued in [6] in this limit one gets on the one hand SYM and on the other type IIB on some space-time. The only difference between the case in [6, 11], is that the D-branes are wrapped on a compact direction. Indeed the sigma model action for a fundamental string on this background is finite.

If the D-p-branes are not compact they result in a SYM (for $p>1$ ) with a moduli space. The propagation of the Type IIB strings then gives the excitation in the particular vacuum defined by the moduli. If however the D-p-branes are compact then the SYM is more complicated and in particular 
can move on it classical vacuum manifold, which is what is usually calculated in the $\mathrm{M}$ (atrix) theory. The energy dependence of a D-brane configuration is defined in the supergravity side by equation (22), while on the SYM side it corresponds to calculation of the vacuum energy. So there is an identification between vacuum energy and the classical action of a probe in a supergravity background. Indeed the SYM (or supergravity) gives the scattering off the DLCQ background, as one wants in the M(atrix) theory.

The supergravity contains the effect of the D-branes on each other, as well as the effect of perturbations of the background, on the D-branes. Given that both supergravity and SYM description are correct in a certain limit, it means that in the $\mathrm{M}$ (atrix) model one can represent the coupling to the background, as one expects in DLCQ.

The guiding principle to describe the coupling are scattering calculations off black-hole background and the corresponding SYM calculations in Dbrane language. This was exploited in [12, 13] for the infinite D3-brane case. These authors identified the SYM as the theory living on the boundary of $A d S_{5}$. Any perturbation on the AdS background induces a non trivial function on the boundary. The correspondence between the SYM and the supergravity is then

$$
W_{\text {sym }}^{\prime}(f(x))=\ln Z_{\text {string }}(f(x, U))
$$

The generator of connected Greens function $W^{\prime}$ is evaluated using the action

$$
S_{y m}^{\prime}=S_{y m}+\int f(x) \Theta(x)
$$

and $f(x, U)$ are some perturbation around $A d S_{5}$, and $f(x)$ is their value at the boundary of $A d S_{5}$. It is important to note that $f(x)$ has space like momenta [12], and thus is a background

For all other D-brane it is actually known that scattering calculations do not agree. For the D1-brane they can agree in the correspondence point [38], and if one uses the stretched throat they may scale correctly with energy, for all D-branes 39. The absorption cross section for gravitons is computed from the two-point function of the energy-momentum tensor on the SYM side. The disagreement between the supergravity calculations and the SYM calculation can be taken as a sign of large $N$ effects in the SYM 9

Let us view now the calculation of the previous section of scattering of Dbranes in this Language. While the above construction was only conjectured to be true for infinite D3-branes, let us for a moment extend it. If for instance the D3-branes were compact, they could be moved with a finite energy. One

\footnotetext{
${ }^{4}$ I would like to thank I. Klebanov and O. Ganor for discussion on this point

${ }^{5}$ As the geometry is singular, for generic D-branes, it is not clear how trustworthy the gravity calculations are. They can be a better approximation once one looks at the thermal SYM in which case there is a large horizon on the gravity side.
} 
way to calculate the velocity dependent potential is to follow [36, 37. Start with the solution describing static D3-branes. Then take one coordinate $r$ (moduli of the SYM) and write everywhere $r=r+v t$. This is not a solution any more. Then one solves the equation of motion to some order in $v$ and inserts to the classical action. From it one can read off the potential. This is just another example of the general relationship, like equation (26). In the limit where the partition function is dominated by the classical minima the right hand side of equation (26) is just the classical action as a function of some initial or boundary conditions. The SYM gives those boundary (or initial) conditions for solving the classical equations of motion, by specifying a fluctuation $f(x)$, and expectation values of fields (possibly time dependent). In general this would then give a procedure for calculating scattering of D-branes in the presence of some fluctuation, which is a manifestation of an originally non-trivial background. The problem however is that the supergravity solution is not well defined everywhere, and in particular for the D-string is not valid at very large $r$. If there are no fluctuations, instead of solving the classical equations of motion which can be very hard, we have already seen there is a short cut to evaluate the velocity dependent potential. One views one of the groups of D-branes as a background and couple another one as a probe. This is just equation (22). For a certain region of $r$ this gives reliable answers.

Now what if we have a small perturbation above the background. The perturbation can be accounted for in the probe calculation by inserting the perturbed background. Here we are thinking of a perturbation above the background of the target only, not taking into account the probe. We now suggest what this correspond to in the SYM side.

In a similar fashion to [12, 13], we suggest that For each background field (dilaton, graviton, etc) one adds to the SYM action a coupling

$$
S_{y m}^{\prime}=S_{y m}+\int f(x) \Theta(x)
$$

where $f(x)$ is the perturbation of the background evaluated at the probebrane position, and $\Theta$ is an operator in the SYM. For example, for the graviton $\Theta$ is the energy momentum tensor of the SYM. One can get the form of the operator (at least the bosonic part) from the Born-Infeld action, alternatively one can look at scattering in M(atrix) theory and deduce, to leading order in energies, The coupling of a generic state to the massless modes. In the case of D0-brane this was described in 40.

Calculation of the vacuum energy in SYM (properly Higgsed) should now give the result corresponding to the perturbed background. This would hopefully resolve the issues discussed in [41, 42]. Of course as the D-branes move the function $f$ will depend on their position. In more complex situations one might need to use a more exact treatment like in 443, 44]. 


\section{Acknowledgments}

I would like to thank O. Bergman, O. Ganor, D. Kabat, I. Klebanov and S.D. Mathur for helpful discussions.

\section{References}

[1] N. Seiberg Why is the Matrix Model Correct hep-th/9710009.

[2] T. Banks, W. Fischler, S. Shenker and L. Susskind, M-theory as a matrix model: a conjecture, hep-th/9610043.

[3] L. Susskind, Another Conjecture about M(atrix) Theory, hepth/9704080.

[4] A. Sen, D0 Branes on $T^{n}$ and Matrix Theory, hep-th/9709220.

[5] K. Becker, M. Becker, J. Polchinski and A. Tseytlin, Higher Order Graviton Scattering in M(atrix) Theory, hep-th/9706072.

[6] J. Maldacens, The Large N Limit of Superconformal Field Theories and Supergravity, hep-th/9711200.

[7] J. Maldacena and A. Strominger, Universal low energy dynamics for rotating black holes, hep-th/9702015.

[8] I. Klebanov, world volume approach to absorption by nondilatonic branes, hep-th/9702076.

[9] S. Gubser, I.Klebanov and A.A. Tseytlin, string theory and classical absorption by three-branes, hep-th/9703040.

[10] S. Gubser and I. Klebanov, Absorption by branes and Schwinger terms in the world volume theroy, hep-th/9708005.

[11] N. Itzhaki, J. Maldacena, J. Sonnenschein and S. Yankielowicz, Supergravity and The Large $N$ Limit of Theories with Sixteen Supercharges, hep-th/9802042.

[12] S.S. Gubser, I.R. Klebanov and A.M. Polyakov, Gauge Theory Correlators from Non-Critical string Theory, hep-th/9802109.

[13] E. Witten, Anti De-Sitter Space and Holography, hep-th/9802150.

[14] S. Hellerman and J. Polchinski Compactification in the Lightlike Limit, hep-th/9711037.

[15] A. Bilal, A comment on compactification of M-theory on an (almost) light-like circle, hep-th/9801047. 
[16] L. Motl, proposals on Non-perturbative Superstring Interactions, hepth/9701025.

[17] T. Banks and N. Seiberg, Strings from Matrices, hep-th/9702187.

[18] R. Dijkgraaf, E. Verlinde and H. Verlinde, Matrix string theory, hepth/9703030.

[19] S. Das and S.D. Mathur Excitation of D-strings, Entropy and Duality, hep-th/9601152.

[20] M.R. Douglas, D. Kabat, P. Pouliot and S. Shenker, D-branes and short distances in string theory, hep-th/9608024.

[21] E. Witten On the Conformal Field theory of the Higgs Branch, hepth/9707093.

[22] O. Aharony, M. Berkooz, S. Kachru, N. Seiberg and E. Silverstein, Matrix Description of Interacting Theories in Six dimensions, hepth/9707079.

[23] A. Hanany and G. Lifschytz, M(atrix) theory on $T^{6}$ and a $m$ (atrix) theory description of KK monopoles, hep-th/9708037.

[24] M.B. Green, J.H. Schwarz and E. Witten, Superstring Theory, Cambridge University Press, Cambridge 1986.

[25] K. Becker, M. Becker, On Graviton Scattering Amplitudes in M-Theory, hep-th/9712238.

[26] E. Keski-Vakkuri and P. Kraus, Short Distance Contributions to Graviton-Graviton Scattering: Matrix Theory versus Supergravity, hepth/9712013.

[27] M. Dine and A. Rajaraman, Multigraviton Scattering in Matrix Model, hep-th/9710174.

[28] G.E. Arutyunov and S.A. Frolov, Four graviton scattering amplitude from $S^{N} R^{8}$ supersymmetric orbifold sigma model, hep-th/9712061.

[29] V. Balasubramanian, R. Gopakumar and F. Larsen, Gauge theory, Geomentry and the Large N Limit, hep-th/9712077.

[30] E. Bergshoeff, C. Hull and T. Ortin, Duality in the Type-II superstring effective action, hep-th/9504081.

[31] I. Chepelev and A.A. Tseytlin, Long distance interaction of branes: correspondence between supergravity and super Yang-Mills description, hep-th/9709087. 
[32] E. Keski-Vakkuri and P. Kraus, Born-Infeld action from Matrix theory, hep-th/9709122.

[33] S. Hyun, The Background Geometry of DLCQ supergravity, hepth/9802026.

[34] H.J. Boonstra, B. Peeters and K. Skenderis, Duality and Asymptotic Geometries, hep-th/9706192.

[35] G.T. Horowitz and A.R. Steif, Phys. Rev. Lett 64 (1990) 260.

[36] N.S. Manton, Phys. Lett. B110 (1982) 54; Phys. Lett. B154 (1985) 397; Phys. Lett. B157 (1985) 475.

[37] R.C. Ferrell and D.M. Eardley, Phys. Rev. Lett. 59 (1987) 1617.

[38] S. Das, Emission from parallel p-brane Black Holes, hep-th/9705165.

[39] R. Emparan, Absorption of Scalars by Extended Objects, hepth/9706204.

[40] D. Kabat and W. Taylor Linearized supergravity from Matrix theory, hep-th/9712185

[41] M.R. Douglas, H. Ooguri and S.H. Shenker, Issues in M(atrix) theory compactification, hep-th/9702203.

[42] M.R. Douglas and H. Ooguri, Why is Matrix theory hard, hepth/9710178.

[43] M.R. Douglas D-branes in Curved Space, hep-th/9703056; D-branes and Matrix theory in Curved Space, hep-th/9707228.

[44] B. de Wit, K. Peeters, and J. Plefka, Supermembranes and Supermatrix Models, hep-th/9712082. 